Proceedings of 6th Workshop on Quantum Chaos and Localisation Phenomena, Warsaw, May 24-26, 2013

\title{
Isoscattering Microwave Networks — The Role of the Boundary Conditions
}

\author{
M. EAWNICZAK ${ }^{a}, \mathrm{~S} . \mathrm{BAUCH}^{a}$, A. SAWICKI ${ }^{b, c}, \mathrm{M} \mathrm{KUS}^{b}$ AND L. SIRKO ${ }^{a}$ \\ ${ }^{a}$ Institute of Physics, Polish Academy of Sciences, al. Lotników 32/46, 02-668 Warszawa, Poland \\ ${ }^{b}$ Center for Theoretical Physics, Polish Academy of Sciences, al. Lotników 32/46, 02-668 Warszawa, Poland \\ ${ }^{c}$ School of Mathematics, University of Bristol, University Walk, Bristol BS8 1TW, UK
}

\begin{abstract}
The recent paper by Hul et al. (Phys. Rev. Lett. 109, 040402 (2012), see Ref. [7]) addresses an important mathematical problem whether scattering properties of wave systems are uniquely connected to their shapes? The analysis of the isoscattering microwave networks presented in this paper indicates a negative answer to this question. In this paper the sensitivity of the spectral properties of the networks to boundary conditions is tested. We show that the choice of the proper boundary conditions is extremely important in the construction of the isoscattering networks.
\end{abstract}

DOI: $10.12693 /$ APhysPolA.124.1078

PACS: 03.65.Nk, 05.45.Ac

\section{Introduction}

More than 25 years were necessary to solve the problem expressed in the famous question "Can one hear the shape of a drum?", posed by Marc Kac in 1966 [1]. It concerns the problem of uniqueness of the spectrum of the Laplace operator on a planar domain with the Dirichlet boundary conditions. The theoretical solution, indicating that in general the spectrum is not unique, was found in 1992 [2] and confirmed experimentally, using microwave cavities, two years later [3]. Similar problems were also considered in the context of quantum graphs [4]. For example, the question regarding the possibility of the determination of the geometry of a quantum graph in the scattering experiment was, on the basis of the theoretical considerations, recently answered in the negative $[5,6]$.

We present here the results of the scattering experiment on microwave isoscattering networks simulating quantum isoscattering graphs where these theoretical predictions were tested. The experimental results were obtained for the frequency range $0.01-2 \mathrm{GHz}$ which is broader than the one demonstrated in [7]. Furthermore, we tested the influence of the boundary conditions on the construction of the isoscattering networks showing their crucial role.

\section{Experiment}

Quantum graphs can be considered as idealizations of physical networks in the limit where the diameters of the wires are much smaller than their lengths. They were successfully applied to model variety of physical problems, see, e.g., [8] and references cited therein. They can also be realized experimentally. Recent developments in various epitaxy techniques allowed also for the fabrication and design of quantum nanowire networks [9, 10]. In $2004 \mathrm{Hul}$ et al. [11] showed that quantum graphs could be successfully simulated by microwave networks. The experimental confirmation of the existence of isoscattering networks simulating isoscattering graphs was demonstrated in a recent paper by $\mathrm{Hul}$ et al. [7]. A microwave network is a structure of $N$ vertices connected by $B$ bonds, e.g., coaxial cables. Each vertex $i$ of a network is connected to the other vertices by $v_{i}$ bonds, $v_{i}$ is called the valency of the vertex $i$. Here we consider networks with typical physical vertex boundary conditions, the Neumann and Dirichlet ones. The first one imposes the continuity of waves propagating in bonds meeting at $i$ and vanishing of the sum of their derivatives calculated at the vertex $i$. The latter demands vanishing of the waves at a vertex. A coaxial cable consists of an inner conductor of radius $r_{1}$ surrounded by a concentric conductor of inner radius $r_{2}$. The space between the inner and the outer conductors is filled with a homogeneous material having the dielectric constant $\varepsilon$.

Below the onset of the next $\mathrm{TE}_{11}$ mode [12], inside a coaxial cable only the fundamental TEM mode can propagate, in the literature often called a Lecher wave. We use the continuity equation for the charge and the current to find the propagation of a Lecher wave inside the coaxial cable joining the $i$-th and the $j$-th vertex of the microwave network $[11,13]$ :

$$
\frac{\mathrm{d}^{2}}{\mathrm{~d} x^{2}} U_{i j}(x)+\frac{\omega^{2} \varepsilon}{c^{2}} U_{i j}(x)=0,
$$

where $e_{i j}(x, t)$ and $J_{i j}(x, t)$ are the charge and the current per unit length on the surface of the inner conductor of a coaxial cable. The potential difference $U_{i j}(x, t)$ between the conductors of a cable is given by

$$
U_{i j}(x, t)=V_{2}^{i j}(x, t)-V_{1}^{i j}(x, t)=\frac{e_{i j}(x, t)}{\mathcal{C}},
$$

where $V_{1}^{i j}(x, t)$ and $V_{2}^{i j}(x, t)$ are the potentials of the inner and the outer conductors of a coaxial cable and $\mathcal{C}$ is the capacitance per unit length of a cable. The spatial derivative of (2) gives [13]: 


$$
\frac{\mathrm{d}}{\mathrm{d} x} U_{i j}(x)=-Z J_{i j}(x),
$$

where $Z$ is the impedance per unit length. Calculation of the second spatial derivative of $U_{i j}(x, t)$ leads to the equation

$$
\frac{\mathrm{d}^{2}}{\mathrm{~d} x^{2}} U_{i j}(x)+Z \frac{\mathrm{d}}{\mathrm{d} x} J_{i j}(x)=0 .
$$

Using Eqs. (1) and (2), Eq. (4) can be transformed to

$$
\frac{\mathrm{d}^{2}}{\mathrm{~d} x^{2}} U_{i j}(x)-Z \mathcal{C} \frac{\mathrm{d}}{\mathrm{d} x} U_{i j}(x)=0 \text {. }
$$

For a monochromatic wave propagating along the cable $e_{i j}(x, t)=\exp (-\mathrm{i} \omega t) e_{i j}(x)$ and $U_{i j}(x, t)=$ $\exp (-\mathrm{i} \omega t) U_{i j}(x)$, where the angular frequency $\omega=2 \pi \nu$ and $\nu$ is the microwave frequency. The impedance per unit length is in this case $Z=R-\mathrm{i} \omega L / c^{2}$, where $R$ and $L$ denote the resistance and the inductance per unit length, respectively, and $c$ is the speed of light in a vacuum. For an ideal lossless cable with the resistance $R=0$ Eq. (5) leads to the telegraph equation on the microwave network

$$
\frac{\mathrm{d}^{2}}{\mathrm{~d} x^{2}} U_{i j}(x)+\frac{\omega^{2} \varepsilon}{c^{2}} U_{i j}(x)=0,
$$

where $\varepsilon=L C$ [14]. The continuity equation for the potential difference requires that for every $i=1, \ldots, N$

$$
\begin{aligned}
& \left.U_{i j}(x)\right|_{x=0}=\varphi_{i},\left.\quad U_{i j}(x)\right|_{x=L_{i j}}=\varphi_{j}, \\
& i<j, \quad C_{i j} \neq 0 .
\end{aligned}
$$

$L_{i j}$ represents the length of the bond joining the $i$-th and the $j$-th vertex of the network. The element $C_{i j}$ of the $N \times N$ connectivity matrix of a network takes the value 1 if the vertices $i$ and $j$ are connected and 0 otherwise. The current conservation condition imposing the Neumann vertex boundary condition may be written in the form

$$
-\left.\sum_{j<i} C_{i j} \frac{\mathrm{d}}{\mathrm{d} x} U_{i j}(x)\right|_{x=L_{i j}}+\left.\sum_{j>i} C_{i j} \frac{\mathrm{d}}{\mathrm{d} x} U_{i j}(x)\right|_{x=0}=0,
$$

where

$$
\frac{\mathrm{d}}{\mathrm{d} x} U_{i j}(x)=Z J_{i j}(x),
$$

Assuming the following correspondence: $\Psi_{i j}(x) \leftrightarrow$ $U_{i j}(x)$ and $k^{2} \leftrightarrow \frac{\omega^{2} \varepsilon}{c^{2}}$, Eq. (6) is formally equivalent to the one-dimensional Schrödinger equation (with $\hbar=$ $2 m=1$ ) on the graph possessing time reversal symmetry [15]:

$$
\frac{\mathrm{d}^{2}}{\mathrm{~d} x^{2}} \Psi_{i j}(x)+k^{2} \Psi_{i j}(x)=0 .
$$

Moreover, Eqs. (7) and (8) are equivalent to the equations derived in [15] for quantum graphs with the Neumann boundary conditions.

Various spectral and scattering properties of microwave networks simulating quantum graphs have been studied so far $[11,16-20]$. One should point out that the introduction of one-dimensional microwave networks simulating quantum graphs increases greatly the number of systems which are used to verify wave effects predicted on the basis of quantum physics [21-27]. The other sys- tems used for this purpose include e.g., two-dimensional and three-dimensional microwave chaotic billiards and experiments with highly excited hydrogen and helium atoms. Experiments for two-dimensional microwave systems were pioneered by [28] and further developed by [29-40]. Three-dimensional chaotic microwave billiards have been also studied experimentally [41-44]. However, for these systems there is no direct analogy between the vectorial Helmholtz equation and the Schrödinger one.

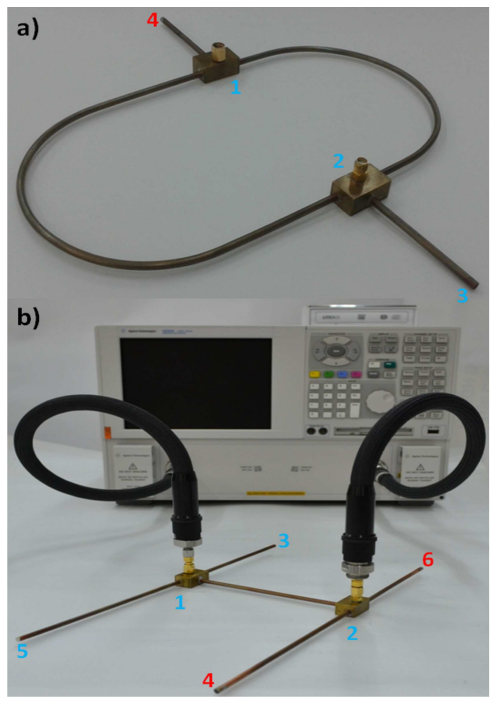

Fig. 1. The network No. I with $N=4$ vertices and the network No. II $(N=6)$ connected to VNA, parts (a) and (b), respectively. The blue numbers denote vertices with the Neumann boundary condition. The vertices with the Dirichlet boundary condition are denoted by the red numbers.

In order to verify experimentally a negative answer to the modified Mark Kac's question we consider the two microwave networks (Fig. 1) which simulate [11] the two isoscattering graphs. The network No. I consists of four bonds and four vertices, whereas the second one consists of five bonds and six vertices. To obtain the scattering networks two infinite leads (in the experiment two elastic microwave cables) were connected to the vertices 1 and 2 of each network. The optical lengths of the microwave networks, which were obtained by rescaling of the physical lengths by the factor $\sqrt{\varepsilon}$, where $\varepsilon \approx 2.08$ is the dielectric constant of a homogeneous material filling the space between the inner and outer leads of the cables, were the same and had the following value: $1.0504 \pm 0.0010 \mathrm{~m}$. The vertices No. 1 and No. 2, defined by the number of bonds and leads meeting at a given vertex, are four-valent, whereas the remaining vertices are one-valent. The Neumann boundary conditions (continuity of waves and vanishing of their derivatives) were imposed at all vertices except the vertices No. 4 and No. 6 with the Dirichlet boundary conditions (vanishing of waves). Such systems are described by the two port scattering matrix $S(\nu)$ : 


$$
S(\nu)=\left(\begin{array}{ll}
S_{11} & S_{12} \\
S_{21} & S_{22}
\end{array}\right) .
$$

In the case when the networks are isoscattering and dissipative the phases

$$
\log \left(\operatorname{det}\left(S^{\mathrm{I}}(\nu)\right)\right)=\log \left(\operatorname{det}\left(S^{\mathrm{II}}(\nu)\right)\right) .
$$

and the modulus of the determinants (amplitude)

$$
\left[\operatorname{det}\left(S^{\mathrm{I}}(\nu)\right)\right]=\left[\operatorname{det}\left(S^{\mathrm{II}}(\nu)\right)\right]
$$

of their scattering matrices should be equal for all values of frequency. In order to measure the two port scattering matrix $S(\nu)$ the networks were connected to an $\mathrm{Ag}$ ilent E8364B vector network analyzer (VNA) via leads (Fig. 1).

\section{Results}

The moduli and phases of the determinants determined for the microwave network No. I (blue solid line) and No. II (red full circles) in the frequency range $0.01-2 \mathrm{GHz}$ are shown in the parts (a) and (b) of Fig. 2, respectively. The agreement between the results obtained for both networks is excellent which confirms that the networks are isoscattering.

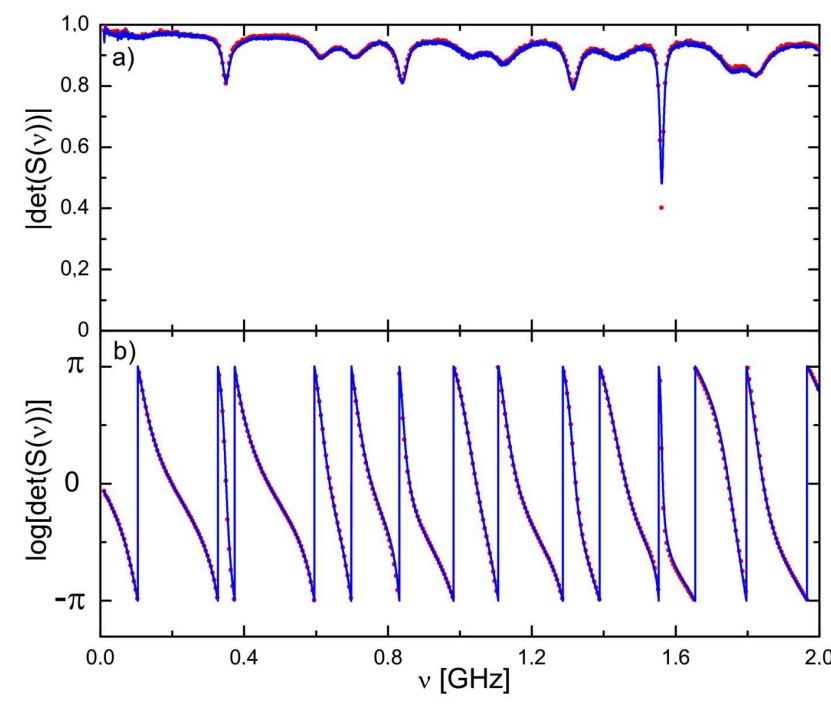

Fig. 2. The amplitude and the phase of the determinant of the scattering matrix obtained for the microwave networks with $N=4$ (blue solid line) and $N=6$ (red full circles) vertices, parts (a) and (b), respectively.

To test the sensitivity of the spectral properties of the networks to boundary conditions, the measurements of the $S(\nu)$ of the networks I and II with the boundary condition of the vertex 3 and 5 , respectively, changed from the Neumann to Dirichlet one were performed. A comparison of the results obtained for the original network I (blue solid line) with the ones obtained for the same network in which the Neumann boundary condition at the vertex 3 was replaced by the Dirichlet one is presented by blue open circles in Fig. 3. In Fig. 3 we also show

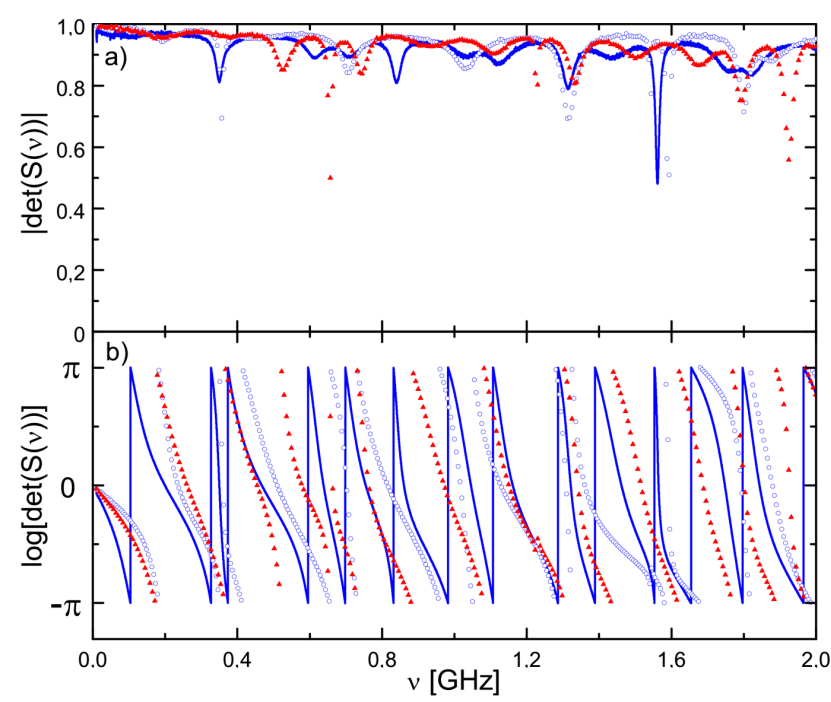

Fig. 3. (a) The amplitude and (b) the phase of the determinant of the scattering matrix obtained for the microwave network with $N=4$ vertices (blue solid line) compared to the results obtained for the same microwave network with the Neumann boundary condition at the vertex 3 replaced by the Dirichlet one (blue open circles). The red full triangles present the results obtained for the network with $N=6$ vertices where the Neumann boundary condition at the vertex 5 was replaced by the Dirichlet one.

the results obtained for the network II with the Neumann boundary condition at the vertex 5 replaced by the Dirichlet one (red full triangles). Our results clearly show that such modifications severely destroy isoscattering features. It should be stressed that the results of the measurements are not only sensitive to the choice of the boundary conditions but they also depend on the accuracy of the preparation of all elements of the microwave networks. The uncertainties of the bond lengths, which limit the frequency range of the measurements, are mainly due to the difficulties in the preparation of the Neumann boundary condition at the vertices.

\section{Conclusions}

We presented the results confirming the theoretical prediction on the impossibility of the determination of the geometry of a quantum graph in the scattering experiment. We also showed that modifications of the boundary conditions of the networks severely destroy their isoscattering features. It is worth to mention that microwave networks can be successfully used to investigate properties of any quantum graphs, also with highly complicated topology, showing a great research potential of quantum simulations based on microwave systems.

\section{Acknowledgments}

This work was partially supported by the Ministry of Science and Higher Education grant No. N N202 130239. 


\section{References}

[1] M. Kac, Am. Math. Mon. 73, 1 (1966).

[2] C. Gordon, D. Webb, S. Wolpert, Bull. Am. Math. Soc. 27, 134 (1992).

[3] S. Sridhar, A. Kudrolli, Phys. Rev. Lett. 72, 2175 (1994).

[4] B. Gutkin, U. Smilansky, J. Phys. A 34, 6061 (2001).

[5] J. Boman, P. Kurasov, Adv. Appl. Math. 35, 58 (2005).

[6] R. Band, A. Sawicki, U. Smilansky, J. Phys. A 43, 415201 (2010).

[7] O. Hul, M. Ławniczak, S. Bauch, A. Sawicki, M. Kuś, L. Sirko, Phys. Rev. Lett. 109, 040402 (2012).

[8] S. Gnutzmann, U. Smilansky, Adv. Phys. 55, 527 (2006).

[9] K.A. Dick, K. Deppert, M.W. Larsson, T. Märtensson, W. Seifert, L.R. Wallenberg, L. Samuelson, $N a-$ ture Mater. 3, 380 (2004).

[10] K. Heo, E. Cho, J.E. Yang, Nano Lett. 8, 4523 (2008).

[11] O. Hul, S. Bauch, P. Pakonski, N. Savytskyy, K. Życzkowski, L. Sirko, Phys. Rev. E 69, 056205 (2004).

[12] D.S. Jones, Theory of Electromagnetism, Pergamon Press, Oxford 1964.

[13] L.D. Landau, E.M. Lifshitz, Electrodynamics of Continuous Media, Pergamon Press, Oxford 1960.

[14] G. Goubau, Electromagnetic Waveguides and Cavities, Pergamon Press, Oxford 1961.

[15] T. Kottos, U. Smilansky, Ann. Phys. 274, 76 (1999).

[16] O. Hul, O. Tymoshchuk, Sz. Bauch, P.M. Koch, L. Sirko, J. Phys. A 38, 10489 (2005).

[17] M. Ławniczak, O. Hul, S. Bauch, P. Šeba, L. Sirko, Phys. Rev. E 77, 056210 (2008).

[18] M. Ławniczak, S. Bauch, O. Hul, L. Sirko, Phys. Scr. T 135, 014050 (2009).

[19] M. Ławniczak, O. Hul, S. Bauch, L. Sirko, Acta Phys. Pol. A 116, 749 (2009).

[20] M. Ławniczak, S. Bauch, O. Hul, L. Sirko, Phys. Rev. E 81, 046204 (2010).

[21] R. Blümel, A. Buchleitner, R. Graham, L. Sirko, U. Smilansky, H. Walther, Phys. Rev. A 44, 4521 (1991).

[22] M. Bellermann, T. Bergemann, A. Haffmann, P.M. Koch, L. Sirko, Phys. Rev. A 46, 5836 (1992).

[23] L. Sirko, S. Yoakum, A. Haffmans, P.M. Koch, Phys. Rev. A 47, R782 (1993).
[24] L. Sirko, P.M. Koch, Appl. Phys. B 60, S195 (1995).

[25] L. Sirko, A. Haffmans, M.R.W. Bellermann, P.M. Koch, Europhys. Lett. 33, 181 (1996).

[26] L. Sirko, S.A. Zelazny, P.M. Koch, Phys. Rev. Lett. 87, 043002 (2001).

[27] L. Sirko, P.M. Koch, Phys. Rev. Lett. 89, 274101 (2002).

[28] H.J. Stöckmann, J. Stein, Phys. Rev. Lett. 64, 2215 (1990).

[29] S. Sridhar, Phys. Rev. Lett. 67, 785 (1991).

[30] H. Alt, H.-D. Gräf, H.L. Harner, R. Hofferbert, H. Lengeler, A. Richter, P. Schardt, A. Weidenmüller, Phys. Rev. Lett. 74, 62 (1995).

[31] P. So, S.M. Anlage, E. Ott, R.N. Oerter, Phys. Rev Lett. 74, 2662 (1995).

[32] U. Stoffregen, J. Stein, H.J. Stöckmann, M. Kus, F. Haake, Phys. Rev. Lett. 74, 2666 (1995).

[33] F. Haake, M. Kus, P. Šeba, H.J. Stöckmann, U. Stoffregen, J. Phys. A 29, 5745 (1996).

[34] L. Sirko, P.M. Koch, R. Blümel, Phys. Rev. Lett. 78, 2940 (1997).

[35] S. Bauch, A. Błędowski, L. Sirko, P.M. Koch, R. Blümel, Phys. Rev. E 57, 304 (1998).

[36] L. Sirko, Sz. Bauch, Y. Hlushchuk, P.M. Koch, R. Blümel, M. Barth, U. Kuhl, H.-J. Stöckmann, Phys. Lett. A 266, 331 (2000).

[37] N. Savytskyy, A. Kohler, Sz. Bauch, R. Blümel, L. Sirko, Phys. Rev. E 6403, 6211 (2001).

[38] R. Blümel, P.M. Koch, L. Sirko, Found. Phys. 31, 269 (2001).

[39] Y. Hlushchuk, L. Sirko, U. Kuhl, M. Barth, H.-J. Stöckmann, Phys. Rev. E 63, 046208 (2001).

[40] N. Savytskyy, O. Hul, L. Sirko, Phys. Rev. E 70, 056209 (2004).

[41] S. Deus, P.M. Koch, L. Sirko, Phys. Rev. E 52, 1146 (1995).

[42] U. Dörr, H.J. Stöckmann, M. Barth, U. Kuhl, Phys. Rev. Lett. 80, 1030 (1998).

[43] C. Dembowski, B. Dietz, H.-D. Gräf, A. Heine, T. Papenbrock, A. Richter, C. Richter, Phys. Rev. Lett. 89, 064101-1 (2002).

[44] O. Tymoshchuk, N. Savytskyy, O. Hul, S. Bauch, L. Sirko, Phys. Rev. E 75, 037202 (2007). 